\title{
Applicability of the Richardson method in a complex-energy basis: Fermionic case
}

\author{
O. Civitarese, ${ }^{1}$ M. Reboiro, ${ }^{1}$ and D. Tielas ${ }^{1,2}$ \\ ${ }^{1}$ Department of Physics, University of La Plata. C.C.67 (1900), La Plata, Argentina \\ ${ }^{2}$ Faculty of Engineering, University of La Plata La Plata, Argentina \\ (Received 17 October 2016; revised manuscript received 9 March 2017; published 10 May 2017)
}

\begin{abstract}
We are testing the applicability of the algebraic procedure developed by Richardson for bound states to solve the pairing-force problem in presence of states with complex energies. This scenario, in which nucleons occupy single-particle states with complex energies and interact via the pairing force, is closely related to the microscopic description of nuclei with a large neutron (or proton) excess in basis which includes single-particle resonances. It is shown that the method gives results which are in good agreement with exact solutions. This finding is in coincidence with previously published works, by other authors, based on different methods to describe resonances.
\end{abstract}

DOI: 10.1103/PhysRevC.95.054307

\section{INTRODUCTION}

The experimental search of neutron- or proton-rich nuclei [1] and the subsequent theoretical interpretation of their properties has been in focus over the last decades. From the theoretical side, the need to include the continuum in the single-particle spectrum has become evident, particularly after the pioneering work of Bergreen [2] and Liotta et al. at Stockholm $[3,4]$. These notions have been extended later on by other authors [5-8], and they are by now well-established concepts. The microscopic description of mean-field and pairing effects in the presence of single-particle resonances is a rather difficult task due to the appearance of convergence problems and to the introduction of energy truncations [9]. The treatment of pairing in the presence of single-particle states with complex energies was analyzed by using extensions of the Green's function formalism in Ref. [10] and by using extensions of the BCS approach [11]. In view of these pieces of information, we aim at testing another approach, which has been known for decades, to find solutions to the pairing force problem. This method, formulated by Richardson [12], has recently been applied, rather successfully, to a variety of many-body systems by Dukelski et al. [13-15]. It proved to be particularly useful for the calculation of pairing parameters in a large basis. In this work we adopt the description of resonant states of Ref. [16] and apply Richardson's method to find solutions to the pairing-force problem for fermions moving in a basis which includes such states. Previous attempts in the same direction are documented in papers by Hasegawa and Kaneko [17], where the effects of resonant single-particle states on the pairing correlations are investigated by an exact treatment of the pairing Hamiltonian on the Gamow shell-model basis [3,4]. The authors of Ref. [17] have shown that many-body correlations between bound and resonant particle pairs are indeed important, and they particularly stressed the point that their approach using the Richardson equations is not adequate to this problem. In another work, IdBetan [18] addressed the question of whether one can use the Hamiltonian with constant pairing strength for correlations in the continuum, and generalized the Richardson exact solution for the pairing Hamiltonian including these correlations. In Ref. [18] the continuum is accounted for by introducing a continuation on the real axis and performing a Cauchy integration. There it is concluded that energy levels can be calculated with constant pairing in the continuum [18].

In this paper, and to determine the validity of Richardson's approach under more general conditions, we compare the results obtained with that approach with exact solutions in model spaces which include bound and resonant states. The procedure to obtain exact solutions is based on the extension of the formalism of Ref. [19] to a complex single-particle basis. We explicitly compare real and imaginary parts of the eigenvalues and explore the validity of the approach in presence of bifurcation points. The systematic exploration of the applicability of Richardson's method, which we are presenting here, complements the information provided by the investigations of Refs. [17,18] and, in our opinion, gives ground to the use of the method in an extended complex basis.

In Sec. II we are briefly presenting the essentials of Richardson's method [12] and define the basis in which we are solving the pairing force problem. The results are presented and discussed in Sec. III and our conclusions are presented in Sec. IV.

\section{FORMALISM}

The separable monopole pairing Hamiltonian is written

$$
H=\sum_{k} \epsilon_{k} c_{k}^{\dagger} c_{k}+g \sum_{i j} \sqrt{\Omega_{i}} \sqrt{\Omega_{j}} A_{i}^{\dagger} A_{j},
$$

where $c_{k}^{\dagger}\left(c_{k}\right)$ are single-particle creation (annihilation) operators and $A_{k}^{\dagger}\left(A_{k}\right)$ are pair-creation (annihilation) operators which create (annihilate) pairs of particles coupled to zero total angular momentum. The index $k$ represents the set of quantum numbers needed to specify the single-particle orbit, and $g$ is the strength of the pairing interaction. Each of the single-particle orbits has a half-degeneracy $\Omega_{k}=j_{k}+\frac{1}{2}$, with $j_{k}$ being the corresponding angular momentum of the orbit of energy $\epsilon_{k}$. Since we are working with fermions, the operators $c_{k}^{\dagger}, c_{k}$ obey anticommutation rules.

It was shown [19] that exact solutions of Eq. (1) in a basis of two single-particle levels can be obtained by diagonalization 
in the space of coupled pairs,

$$
\left|n_{1}, \ldots, n_{k}, \ldots, n_{l}\right\rangle=\Pi_{i} \frac{A_{i}^{\dagger n_{i}}}{\sqrt{\left[n_{i}\right] !}}|0\rangle,
$$

where $n_{p}=\sum_{i} n_{i}$ is the total number of pairs in the system.

The expression of the matrix elements of Eq. (1) in the basis (2) is the following:

$$
\begin{aligned}
& \left\langle n_{1}^{\prime} \ldots, n_{k}^{\prime}, \ldots, n_{l}^{\prime}|H| n_{1} \ldots, n_{k}, \ldots, n_{l}\right\rangle \\
& \quad=\left(\Pi_{h} \delta_{n_{h}^{\prime} n_{h}}\right) \sum_{k} 2 \epsilon_{k} n_{k}+g \sum_{i j} \sqrt{\Omega_{j}\left[n_{j}-\delta_{i j}+1\right] \Omega_{i}\left[n_{i}\right]}\left(\Pi_{h \neq i, j} \delta_{n_{h}^{\prime} n_{h}}\right) \delta_{n_{j}^{\prime} n_{j}+1-\delta_{i j}} \delta_{n_{i}^{\prime} n_{i}-1+\delta_{i j}} \\
& \quad=\left(\Pi_{h} \delta_{n_{h}^{\prime} n_{h}}\right) \sum_{k} 2 \epsilon_{k} n_{k}+g \sum_{i j} \sqrt{\left(n_{j}-\delta_{i j}+1\right)\left(\Omega_{j}-n_{j}+\delta_{i j}\right) n_{i}\left(\Omega_{i}-n_{i}+1\right)}\left(\Pi_{h \neq i, j} \delta_{n_{h}^{\prime} n_{h}}\right) \delta_{n_{j}^{\prime} n_{j}+1-\delta_{i j}} \delta_{n_{i}^{\prime} n_{i}-1+\delta_{i j}} .
\end{aligned}
$$

The Richardson equations have the form [12]

$$
\frac{1}{2 g}=\sum_{k=1}^{N_{l}} \frac{d_{k}}{2 \epsilon_{k}-x_{\alpha}}+\sum_{\beta \neq \alpha} \frac{1}{x_{\alpha}-x_{\beta}},
$$

where $N_{l}$ is the number of single-particle levels, $x_{\alpha}$ with $\alpha=$ $1,2, \ldots, n_{p}, n_{p}$ being the number of pairs, are the complexvalue parameters associated with each Richardson pair, and $d_{k}=v_{k}-\Omega_{k} / 2$ is the effective degeneracy of orbit $k$ with seniority $v_{k}$.

In the Richardson method, the eigenvalues of the Hamiltonian are given by the sum of the $x_{\alpha}$ parameters associated with each state and, for seniority-zero states, they are expressed by the sum

$$
E(\{x\})=\sum_{\alpha} x_{\alpha}
$$

for each state, and they are purely real values; that is, the sum of the imaginary part of the set of parameters $x_{\alpha}$ vanishes. The determination of the parameters $x_{\alpha}$ was a matter of algebraic manipulations until Dukelski et al. [13-15] developed a less ambiguous way of finding them. In the present case we solve a system of $n_{p}$ coupled equations in the variables $x_{\alpha}$. Each equation of the system has the form [12]. As said before, the correspondence between the eigenvalues of the Hamiltonian (1) and the set of parameters $x_{\alpha}$ is unique, but in some situations, which we illustrate in the next section, it may not be so clearly established because of the appearance of bifurcations and level crossings. This effect may be even more manifest if the single-particle basis has states with complex energies, and this is the point we want to investigate since it may be crucial for the stability of the solutions of Richardson's method.

\section{RESULTS AND DISCUSSIONS}

We present, first, the results obtained by an exact diagonalization of the Hamiltonian, in a two-level basis, where the energy level of the upper shell will be taken as real, and afterwards taken as complex, in order to grasp the main features of the solutions. Figures 1 and 2 show the exact solutions and those obtained with Richardson's method, respectively, for a system of three pairs interacting via the pairing force and moving in a space of two shells with degeneracy $\Omega$. The energies and Richardson's parameters are given as functions of the variable $g \Omega / 2 \epsilon$, which is the ratio between the pair interaction energy and the energy spacing of the two levels. For this case, we have taken all energies as real. The configuration space is spanned by four states, corresponding to the four configurations of three pairs moving in two levels.

The exact solutions are well separated and the lowest one shows the typical behavior due to the pairing interaction in the space of pairs, which is the building up of coherent correlations which push down the energy of one of the states [19]. Since all configurations have real energies, all of the eigenvalues are real.

The results of Fig. 2 are consistent with the exact solution. Since we have three pairs the method yields three parameters: $x_{1}, x_{2}, x_{3}$. Two of them are complex-conjugate quantities and the remaining one is real. The sum of the real part of these parameters, for each of the four states, yields the value of the exact solutions. To help with the comparison we have listed



FIG. 1. Exact eigenvalues for a two-level system with $\Omega_{1}=\Omega_{2}=$ $\Omega=10$, and energies $\epsilon_{1}=\frac{\epsilon}{2}$ and $\epsilon_{2}=-\frac{\epsilon}{2}$ and number of fermion pairs, $n_{p}=3$. Both levels have real energy. The energies are given in arbitrary units. 



FIG. 2. Richardson pair energies $\left(x_{\alpha}\right)$ for the system of Fig. 1. In the insets of the figure, from bottom to top, we show the real (left panel) and imaginary (right panel) components of the solutions for the ground state, and first-, second-, and third-excited states, respectively. The energies are given in arbitrary units.

in Table I the exact solutions and the values of the associated Richardson parameters for a given value of the ratio $g \Omega / 2 \epsilon$.

After showing that the determination of Richardson's parameters for three pairs of fermions, interacting in a basis consisting of two levels with real energy and the same degeneracy, is indeed feasible and that it yields the same values as the exact diagonalization of the Hamiltonian in the space of

TABLE I. Numerical values of the exact solutions and Richardson's parameters for the system of three pairs in a two-level system. The parameters of the system are given in the caption to Fig. 1 and the table shows the results obtained with $g \Omega / 2 \epsilon=0.4$. The column denoted by $\lambda_{i}$ indicates the eigenvalue index, the real and imaginary part of the exact eigenvalues are given in the second and third columns, respectively. The Richardson's parameters $x_{\alpha}$ for each eigenstate are given in the fifth (real part) and sixth (imaginary part) columns. All values are given in arbitrary units of energy.

\begin{tabular}{llllllr}
\hline \hline \multicolumn{3}{c}{ Eigenvalue } & & \multicolumn{3}{c}{ Pair energy } \\
\cline { 1 - 4 } \cline { 5 - 6 }$\lambda_{i}$ & Real & Imaginary & & $x_{i}$ & Real & Imaginary \\
\hline 4 & 1.69568 & 0.00000 & & $x_{1}$ & 0.5796 & 0.2551 \\
4 & 1.69568 & 0.00000 & & $x_{2}$ & 0.5796 & -0.2551 \\
4 & 1.69568 & 0.00000 & & $x_{3}$ & 0.5366 & 0.0000 \\
3 & -0.92850 & 0.00000 & & $x_{1}$ & 0.5019 & 0.1567 \\
3 & -0.92850 & 0.00000 & & $x_{2}$ & 0.5019 & -0.1567 \\
3 & -0.92850 & 0.00000 & & $x_{3}$ & -1.9323 & 0.0000 \\
2 & -3.38744 & 0.00000 & & $x_{1}$ & 0.4376 & 0.0000 \\
2 & -3.38744 & 0.00000 & & $x_{2}$ & -1.9125 & 0.2923 \\
2 & -3.38744 & 0.00000 & & $x_{3}$ & -1.9125 & -0.2923 \\
1 & -5.69974 & 0.00000 & & $x_{1}$ & -1.8699 & 0.5190 \\
1 & -5.69974 & 0.00000 & & $x_{2}$ & -1.8699 & -0.5190 \\
1 & -5.69974 & 0.00000 & & $x_{3}$ & -1.9599 & 0.0000 \\
\hline \hline
\end{tabular}

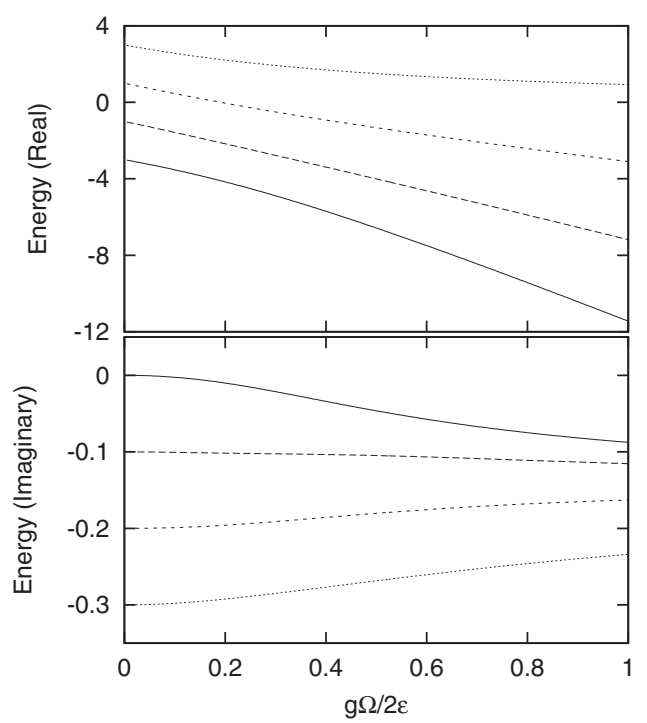

FIG. 3. Exact eigenvalues, for three fermion pairs moving in two levels of degeneracy $\Omega$. The lower level has a real energy $\epsilon_{1}=-\epsilon$ and the upper one has complex energy with real part $\epsilon_{2}=\epsilon$ and imaginary part $\Gamma=-0.05$. All values are given in arbitrary units of energy.

pairs, we repeat the calculation, this time allowing the upper level of the two-level system to have a complex energy. The results of the diagonalization are shown in Fig. 3, both for the real and imaginary part of the eigenvalues. The results which we have obtained by applying Richardson' $s$ method to this case are shown in Fig. 4. As indicated in the captions, $\Gamma$ is the imaginary part of the energy of the upper level of the basis, and for this case the absolute value of the ratio between the

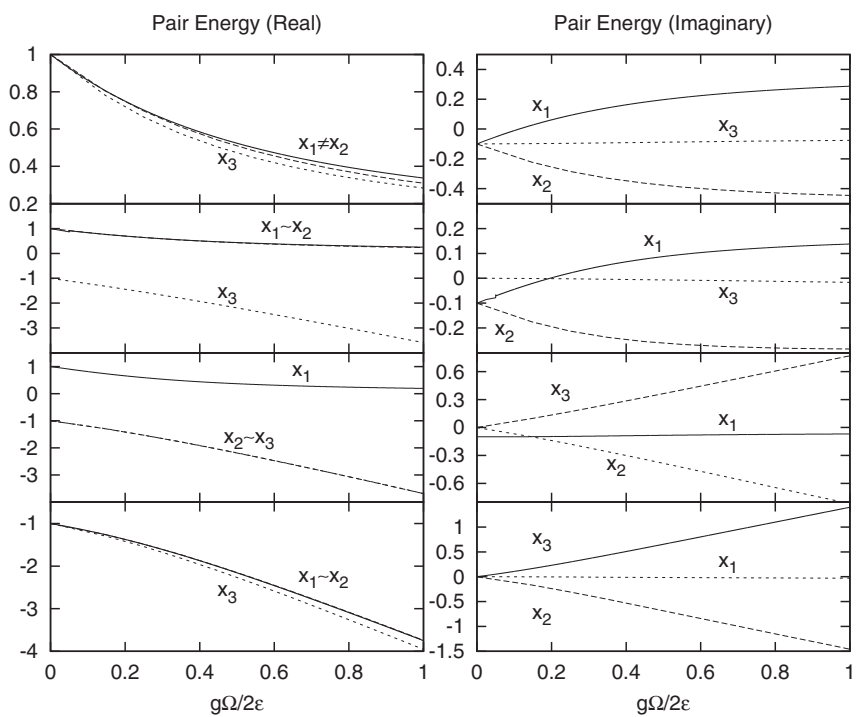

FIG. 4. Richardson pair energies $\left(x_{\alpha}\right)$ for the system of Fig. 3. From bottom to top the insets show the results for the real (left panel) and imaginary (right panel) part of the solutions for the ground state and for the excited states, respectively. 
TABLE II. Exact solutions and values of the Richardson parameters for the pairing eigenvalue problem of three pairs of fermions moving in a two-level basis with the upper level with complex energy, the same of Figs. 3 and 4, and for $g \Omega / 2 \epsilon=0.4$. All values are given in arbitrary units of energy.

\begin{tabular}{|c|c|c|c|c|c|}
\hline \multicolumn{3}{|c|}{ Eigenvalue } & \multicolumn{3}{|c|}{ Pair energy } \\
\hline$\lambda_{i}$ & Real & Imaginary & $x_{i}$ & Real & Imaginary \\
\hline 4 & 1.69484 & -0.27678 & $x_{1}$ & 0.5848 & 0.1625 \\
\hline 4 & 1.69484 & -0.27678 & $x_{2}$ & 0.5738 & -0.3480 \\
\hline 4 & 1.69484 & -0.27678 & $x_{3}$ & 0.5362 & -0.0912 \\
\hline 3 & -0.92905 & -0.18569 & $x_{1}$ & 0.5052 & 0.0663 \\
\hline 3 & -0.92905 & -0.18569 & $x_{2}$ & 0.4978 & -0.2474 \\
\hline 3 & -0.92905 & -0.18569 & $x_{3}$ & -1.9321 & -0.0046 \\
\hline 2 & -3.38733 & -0.10348 & $x_{1}$ & 0.4371 & -0.0883 \\
\hline 2 & -3.38733 & -0.10348 & $x_{2}$ & -1.9106 & -0.2999 \\
\hline 2 & -3.38733 & -0.10348 & $x_{3}$ & -1.9138 & 0.2847 \\
\hline 1 & -5.69846 & -0.03405 & $x_{1}$ & -1.8657 & -0.5303 \\
\hline 1 & -5.69846 & -0.03405 & $x_{2}$ & -1.8733 & 0.5075 \\
\hline 1 & -5.69846 & -0.03405 & $x_{3}$ & -1.9595 & -0.0112 \\
\hline
\end{tabular}

real and imaginary parts of the energy of the upper level is of the order of $2.5 \times 10^{-2}$.

The results shown in Table II are very illustrative of the changes in the structure of the parameters $x_{\alpha}$ for the case of a basis with a complex energy state. The complex-conjugate pairs found for the basis with real energies of Table I are broken and all the eigenvalues now have a complex energy. The values corresponding to the sum of the parameters $x_{\alpha}$ for each configuration agree with the exact eigenvalues obtained from the diagonalization, both in the real and imaginary part of them. The numbers indeed agree quite well, as can be verified by adding, for each eigenvalue $\lambda_{i}$ of Table II, the corresponding Richardson entries $x_{i}$, both for the real and imaginary parts of

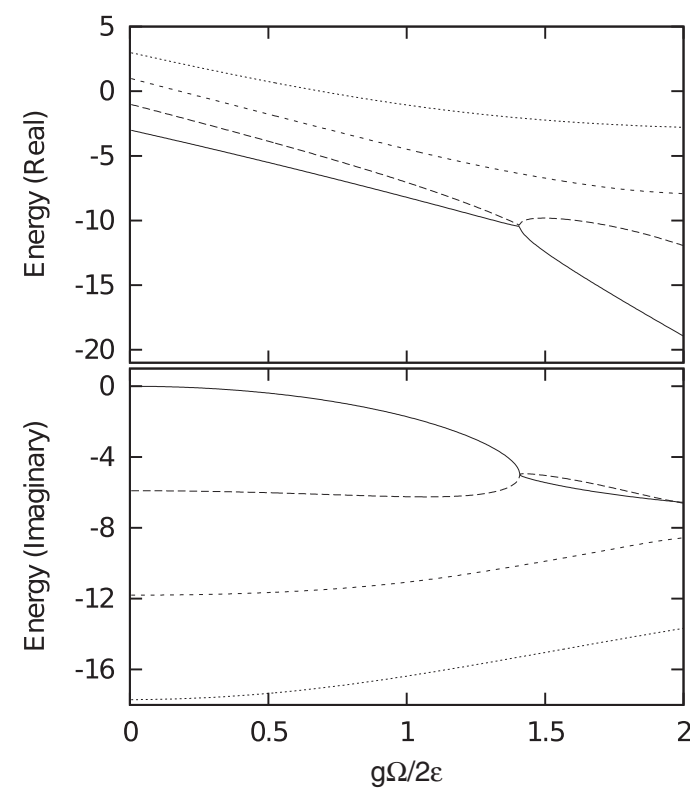

FIG. 5. Exact solutions for the two-level system with $\Gamma=-2.59$, and for the same other parameters given in the caption to Fig. 1.
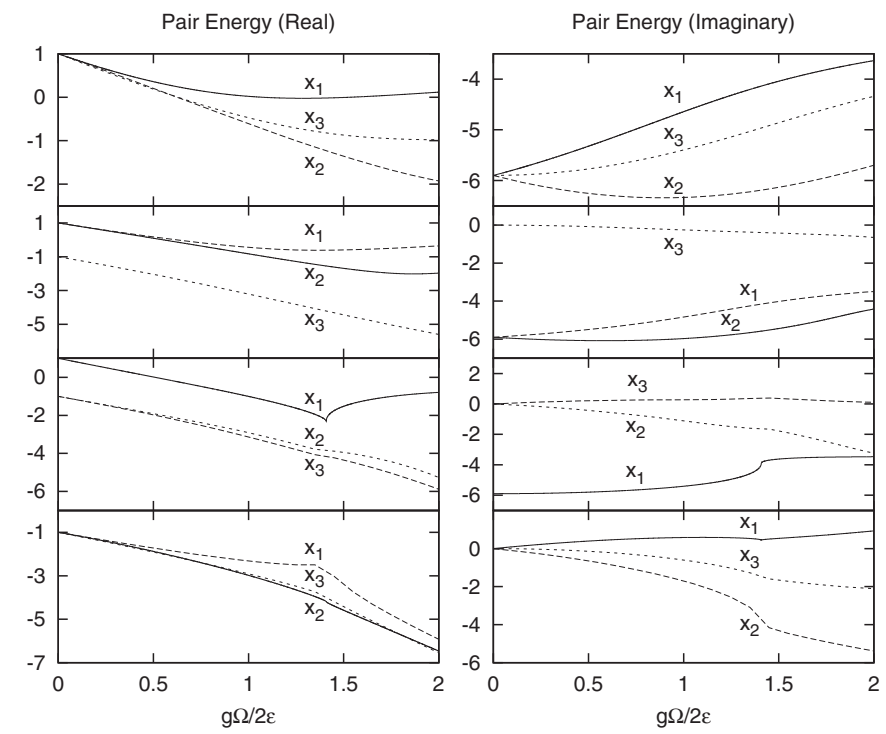

FIG. 6. Real and imaginary parts of the solutions $x_{i}$, for each configuration, for the same case as that of Fig. 5.

them. This is true both for the eigenvalue of lowest energy $\left(\lambda_{i}=1\right.$ of Table II) which has the smaller imaginary part, the ratio between the imaginary and the real part of the solution is of the order of $6 \times 10^{-3}$, and for the eigenvalue with the largest energy $\left(\lambda_{i}=4\right.$ of Table II) where the absolute value of the same ratio is of the order of 0.16 .

The inclusion of complex-energy states in a basis, and the consequences of its use in nuclear structure calculations, as described in Refs. [11] often deals with the question about the presence of broad resonances which may overlap. To investigate the effects of these states on the stability of the solutions, and to complete the numerical analysis of



FIG. 7. Results of the calculations, applying Richardson's method, after adding up the values of $x_{i}$, both real and imaginary, for each eigenvalue. The results shown in this figure are to be compared with the results shown in Fig. 5. 
TABLE III. Numerical values, for the solutions shown in Figs. 5-7, for $g \Omega / 2 \epsilon=0.4$.

\begin{tabular}{llllllr}
\hline \hline \multicolumn{3}{c}{ Eigenvalue } & & \multicolumn{3}{c}{ Pair energy } \\
\cline { 1 - 2 } \cline { 5 - 6 }$\lambda_{i}$ & Real & Imaginary & & $x_{i}$ & Real & Imaginary \\
\hline 4 & 1.17145 & -17.47342 & & $x_{1}$ & 0.4647 & -5.4505 \\
4 & 1.17145 & -17.47342 & & $x_{2}$ & 0.3672 & -6.2057 \\
4 & 1.17145 & -17.47342 & & $x_{3}$ & 0.3396 & -5.8172 \\
3 & -1.22062 & -11.70880 & & $x_{1}$ & 0.3278 & -5.5941 \\
3 & -1.22062 & -11.70880 & & $x_{2}$ & 0.2776 & -6.0615 \\
3 & -1.22062 & -11.70880 & & $x_{3}$ & -1.8261 & -0.0532 \\
2 & -3.27979 & -5.97455 & & $x_{1}$ & 0.2133 & -5.8365 \\
2 & -3.27979 & -5.97455 & & $x_{2}$ & -1.7246 & -0.3192 \\
2 & -3.27979 & -5.97455 & & $x_{3}$ & -1.7685 & 0.1811 \\
1 & -4.99104 & -0.24324 & & $x_{1}$ & -1.6842 & 0.3339 \\
1 & -4.99104 & -0.24324 & & $x_{2}$ & -1.5874 & -0.4903 \\
1 & -4.99104 & -0.24324 & $x_{3}$ & -1.7194 & -0.0869 \\
\hline \hline
\end{tabular}

the applicability of the method, we now discuss the results obtained for the case of a basis where the imaginary part of the upper level is fixed at quite a large value, that is by given to $\Gamma$ a value of the same order of magnitude of the real part, making the absolute value of the ratio between them of the order of unity.

Figure 5 shows the results of the exact diagonalization for the case with $\Gamma=-2.95$, while Fig. 6 shows the solutions, real and imaginary part of $x_{i}$ for each configuration. To make the comparison between the exact results and the Richardson's results more explicit, in Fig. 7 we show the results obtained by adding the values of $x_{i}$ for each eigenvalue, both real and imaginary parts. This last figure shows the appearance of a bifurcation point, and the resulting degeneracy of the some of the solutions after reaching it. The pattern of the bifurcations is quite interesting because it shows a correlation between the real and imaginary part of the eigenvalues, which show a

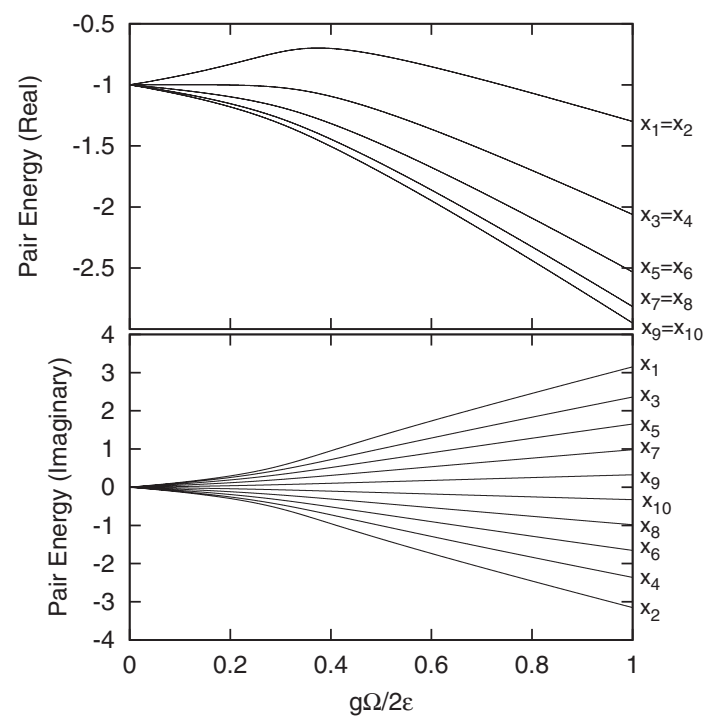

FIG. 8. Richardson method for a two-level system with $\Omega_{i}=10$, $n_{p}=10$. Parameters for the ground state, for the case with $\Gamma=0.0$.

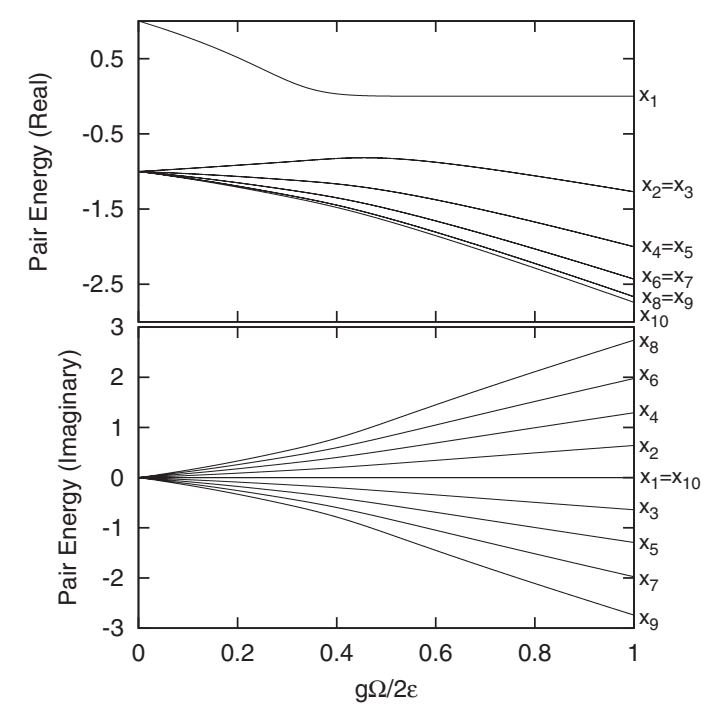

FIG. 9. Richardson method for a two-level system with $\Omega_{i}=10$ and $n_{p}=10$. Parameters $x_{\alpha}$ for the first-excited state, for the case with $\Gamma=0.0$.

pair-like bifurcation; that is, two nearly degenerate real values separate after the bifurcation point and their imaginary parts do the contrary and from well-separated values they converge to a nearly degenerate pair. The bifurcation point for this case is reached at the value $g \Omega / 2 \epsilon=1.409$. This is indeed a characteristic feature of Richardson's solutions, when the imaginary part of the energy of a level (in this case is the upper one of the two-level model space) is comparable to the real part of the energy. In realistic situations this case will correspond to very broad resonances. At this point we should be particularly careful since to a broad single-particle resonance, e.g., a state with a large imaginary part of the energy resulting from the diagonalization in a central potential; for instance, of the type

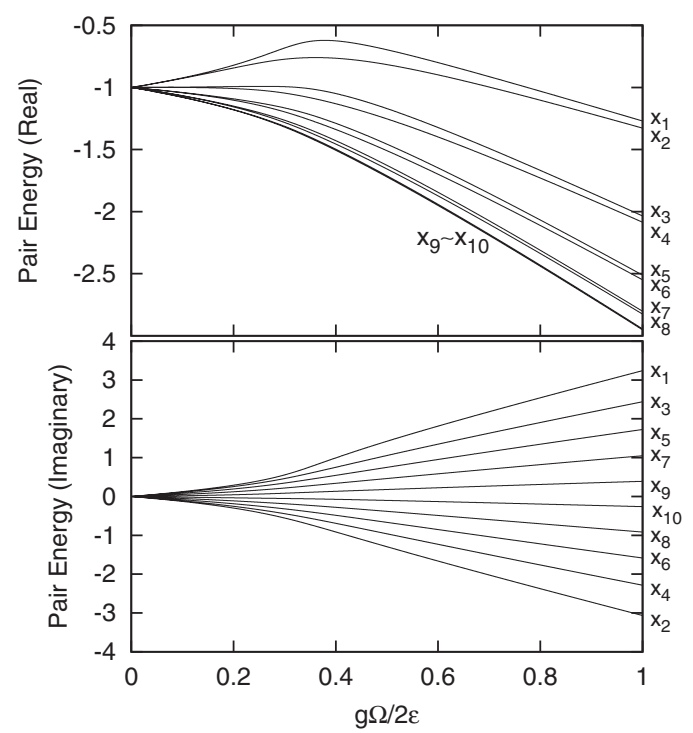

FIG. 10. Richardson method for a two-level system with $\Omega_{i}=$ 10 , and $n_{p}=10$. Parameters for the ground state, for the case with $\Gamma=-0.1$. 




FIG. 11. Richardson method for a two-level system with $\Omega_{i}=$ 10 , and $n_{p}=10$. The curves show the value of the parameters $x_{\alpha}$ of the first-excited state for the case $\Gamma=-0.1$.

used in nuclear structure calculations [4,11], is associated a very spread out radial wave function, and we are using here a separable, radial independent pairing force which does not account for the radial overlaps between states. In spite of this, we remark that the purpose of this part of the calculations is to show the appearance of bifurcations resulting from the presence of a state with a large imaginary part of the energy. An additional limitation for the use of states with a large widths, in the context of Richardson's method, is discussed in Ref. [16], when talking about the occupancies of the single-particle states, whose real parts becomes negative if the width of the state is large.

The numerical solutions corresponding to the curves shown in Figs. 5-7 are given in Table III.

We turn now our attention to cases where the degeneracy of the shells and the number of pairs which can be accommodated becomes larger than in the previous examples, to grasp the dependence of the results upon these variables.

Figure 8 shows the Richardson parameters for the ground state of a system of 10 pairs and $\Omega=10$. Figure 9 shows the results, for the first-excited state for the same system. In both figures the energy of each of the states of the basis is real. In Figs. 10 and 11 we show the results for the same configuration; this time for an upper level with complex energy, with imaginary part $\Gamma=-0.1$. Again in this case, with a large number of pairs and with larger degeneracy, it was possible to find the parameters $x_{\alpha}$ without difficulties.

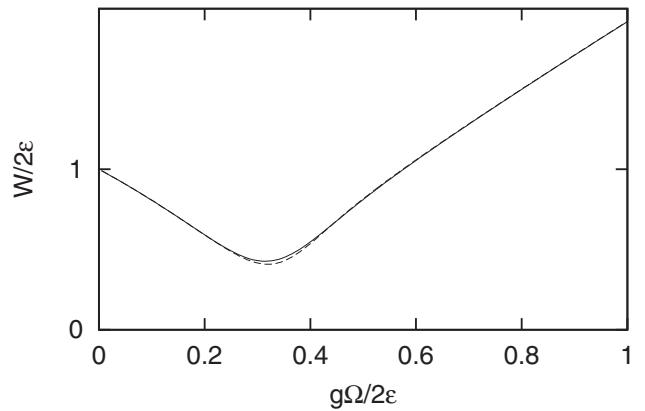

FIG. 12. Real part of the energy difference between the firstexcited state and the ground state, $W=E_{1 \text { st exc. }}-E_{\text {g.s. }}$, obtained by the exact diagonalization and by applying Richardson method, for a two-level system with $\Omega_{i}=10$ and $n_{p}=10$. The results correspond to $\Gamma=0.0$ (continuous line) and $\Gamma=-0.1$ (dashed line).

Finally, let us turn our attention to the behavior of the firstexcited state, this time by drawing the real part of the energy difference between the first-excited state and the ground state. The results, for $\Omega=10, n_{p}=10$, and $\Gamma=0,-0.1$, are shown in Fig. 12. From the curves displayed in this figure, one can see that the appearance of a gap in the spectrum is preserved. Notice that, since the exact results and the results obtained with Richardson method are in fact the same, they cannot be distinguished numerically, therefore the curves show the small difference between the calculations for these values of $\Gamma$.

\section{CONCLUSIONS}

In this work we have presented the results of exact diagonalizations and those obtained by applying Richardson's method to systems of fermions interacting via the pairing force and moving in basis with complex-energy states. The calculations, although restricted to a two-level model space, are demonstrative of the fact that the method due to Richardson can also be applied to a complex energy basis. Since the applicability of Richardson's method to a basis with a large number of states is already known from the work of Dukelski et al. [13-15], its extension to a complex energy basis is indeed legitimate. In this respect the results we have presented complement the findings of Refs. $[17,18]$ where the method was applied without further discussions about its validity and with apparently opposite conclusions.

\section{ACKNOWLEDGMENTS}

The authors are members of the CONICET (Argentina), at the Institute of Physics of La Plata, with grants PIP 282CONICET and PICT 001103 of the ANPCYT.
[1] H. L. Duorah, Nature (London) 232, 247 (1971).

[2] T. Berggren, Nucl. Phys. A 109, 265 (1968); T. Bergren, Phys. Lett. B 373, 1 (1996).

[3] R. Id Betan, R. J. Liotta, N. Sandulescu, and T. Vertse, Phys. Rev. Lett. 89, 042501 (2002).
[4] T. Vertse, P. Curutchet, and R. J. Liotta, Phys. Rev. C 42, 2605 (1990).

[5] N. Michel, W. Nazarewicz, M. Ploszajczak, and K. Bennaceur, Phys. Rev. Lett. 89, 042502 (2002).

[6] A. Volya and V. Zelevinsky, Phys. Rev. Lett. 94, 052501 (2005). 
[7] I. Rotter, Rep. Prog. Phys. 54, 635 (1991).

[8] J. Okołowicz, M. P. Płoszajczak, and I. Rotter, Phys. Rep. 374, 271 (2003).

[9] J. A. Lay, C. E. Alonso, L. Fortunato, and A. Vitturi, J. Phys. G 43, 085103 (2016).

[10] Z. X. Xu, C. Qi, R. J. Liotta, and R. Wyss, J. Phys.: Conf. Ser. 338, 012029 (2012).

[11] N. Sandulescu, O. Civitarese, R. J. Liotta, and T. Vertse, Phys. Rev. C 55, 1250 (1997).

[12] R. W. Richardson, Phys. Lett. 3, 227 (1963); J. Math. Phys. 6, 1034 (1965); Phys. Rev 141, 949 (1966); J. Math. Phys. 9, 1327 (1968); 18, 1802 (1977).
[13] J. M. Roman, G. Sierra, and J. Dukelsky, Phys. Rev. B 67, 064510 (2003).

[14] S. Rombouts, D. Van Neck, and J. Dukelsky, Phys. Rev. C 69, 061303 (2004).

[15] J. Dukelsky, B. Errea, H. S. Lerma, and S. Pittel, Int. J. Mod. Phys. E 16, 210 (2007).

[16] O. Civitarese and M. Gadella, Phys. Rep. 396, 41 (2004)

[17] M. Hasegawa and K. Kaneko, Phys. Rev. C 67, 024304 (2003).

[18] R. Id Betan, Phys. Rev. C 85, 064309 (2012).

[19] J. Högaasen-Feldman, Nucl. Phys. 28, 258 (1961). 